\title{
Utilidad de la determinación de anticuerpos IgG e IgM por ELISA e inmunocaptura en una serie clínica de brucelosis humana
}

\author{
Carolina Aranís J., Jorge Oporto C., Mónica Espinoza, Ingrid Riedel K., Carlos Pérez C. y Patricia García C.
}

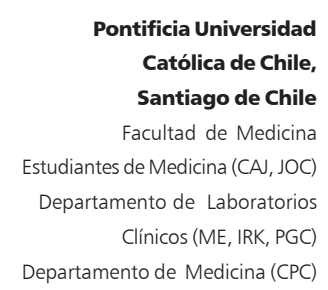

Recibido: 10 de abril de 2007 Aceptado: 7 de noviembre de 2007

Correspondencia a: Patricia García Cañete pgarcia@med.puc.cl

\section{Usefulness of the determination of IgG and IgM antibodies by ELISA and immunocapture in a clinical series of human brucellosis}

Introduction: The diagnostic difficulties of brucellosis makes the evaluation of new diagnostic tests necessary. Objectives: Evaluation of different commercial tests in the serological diagnosis of brucellosis by ELISA and immunocapture antibodies in a clinical series of patients with brucellosis of the Health Network of the Catholic University of Chile. Methods: All the serums received in the Laboratory of Microbiology for suspicion of brucellosis during five years were studied. Two groups were obtained, one that fulfilled diagnostic criteria for brucellosis [clinical evidence, and/or positive blood culture and/or seroagglutination test (SAT) in titers $>1 / 160]$ and the control group. Each serum sample was analyzed using immunocaptureagglutination test (Brucellacapt $\left.{ }^{\circledR}\right)$, ELISA IgM and IgG. Results: Of 10 patients with brucellosis, the serologic results were: $8 / 10$ positives for ELISA IgG, $7 / 10$ for Brucellacapt ${ }^{\circledR}$ and SAT, and 5/10 for ELISA IgM. Discussion: ELISA IgG alone was superior than SAT. The combination ELISA IgG/ Brucellacapt ${ }^{\circledR}$ reaches the best detection performance (9/10) and can be an alternative to SAT.

Key words: Brucellosis, immunology, agglutination test, Brucellacapt ${ }^{\circledR}$.

Palabras clave: Brucelosis, inmunología, aglutinación, Brucellacapt ${ }^{\circledR}$.

\section{Introducción}

B rucelosis es una zoonosis transmisible, siendo Brucella abortus la más importante en nuestro medio. Esta especie afecta al ganado bovino e implica un alto riesgo laboral para veterinarios, personal de mataderos, carniceros y pastores ${ }^{1}$ en quienes la transmisión puede ser por consumo de productos lácteos infectados no pasteurizados, contacto directo entre piel o mucosas lesionadas y animales infectados (sangre, carne o placenta) ${ }^{2}$.

Las zonas afectadas por brucelosis bovina abarcan desde la $\mathrm{IV}^{\circ}$ a XII ${ }^{\circ}$ Región, donde la prevalencia alcanzó en 1991 a 2,4\%3,4 de la masa bovina nacional, luego de 16 años de iniciado el programa de control y erradicación de la enfermedad en 1975, fecha en la que la prevalencia alcanzaba a $7 \%$.

La brucelosis humana es una enfermedad de curso insidioso, que puede derivar en graves complicaciones, por lo que en Chile es de notificación obligatoria inmediata. La prevalencia se desconoce y su incidencia es baja, alcanzando en el año 2003 a 0,06\%, cifra probablemente subestimada; se considera que podría alcanzar tasas de 0,6 a $1,5 \%$, es decir, 10 a 25 veces más alta a las notificadas ${ }^{5,6}$. En ello influyen la gran heterogeneidad de la presentación clínica y la difícil confirmación de laboratorio.

El diagnóstico de certeza es por cultivos (hemocultivo o cultivo de médula ósea), aunque la sensibilidad del método fluctúa entre 15 y 70\% $\%^{7,8}$. Por esta razón, el diagnóstico puede realizarse mediante expresión clínica más serología. Existen diversos métodos de diagnóstico serológico, siendo la seroaglutinación en tubo $(\mathrm{SAT})^{9}$ el estándar de oro por su alta especificidad con títulos $\geq 1 / 160$. Ésta técnica detecta anticuerpos IgG y totales contra antígenos de B. abortus y Brucella melitensis; sin embargo, es laboriosa, utiliza el reactivo 2-mercapto-etanol que es altamente irritante para el personal que lo manipula y requiere 72 horas para la obtención de resultados.

Recientemente han aparecido métodos comerciales como la aglutinación con inmunocaptura (Brucellacapt ${ }^{\circledR}$, VIRCELL SL; Granada, España) ${ }^{10}$ que permite la detección de anticuerpos totales, con una sensibilidad y especificidad de 95 a $100 \%$ y 59 a $95 \%$ respectivamente $^{11}$. Por otro lado, mediante ELISA es posible detectar anticuerpos IgG o IgM anti-lipopolisacarido de $B$. abortus cepa S-99 ${ }^{12}$, existiendo estudios que reportan 
sensibilidades y especificidades de 78 a $98 \%$ y 83 a $98 \%$ para el primero, y 80 a $100 \%$ y 36 a $98 \%$ para el segundo ${ }^{13}{ }^{14,15,16}$. El antígeno utilizado demostró ser útil en la detección de $B$. abortus y $B$. melitensis pero no de Brucella canis y carece de reacciones cruzadas con Salmonella Typhi. Si bien en estudios internacionales SAT sigue siendo eficiente para el serodiagnóstico, se describe que la prueba de ELISA IgG aumentaría el número de casos diagnosticados al combinarse éste y podría ser útil en el seguimiento ${ }^{14}$.

Dado el difícil diagnóstico de esta enfermedad y la reciente aparición de nuevas pruebas diagnósticas aún no validadas en nuestro país, el objetivo de este trabajo fue evaluar la utilidad de tres pruebas serológicas disponibles comercialmente: aglutinación con inmunocaptura (Brucellacapt $\left.{ }^{\circledR}\right)$, ELISA IgM y ELISA IgG, en una serie clínica de brucelosis humana de la Red de Salud de la Pontificia Universidad Católica de Chile.

\section{Pacientes y Métodos}

Se analizaron todos los pacientes con sospecha de brucelosis, cuyos sueros fueron recibidos y almacenados a $-20^{\circ} \mathrm{C}$ en el Laboratorio de Microbiología de la Red de Salud UC para estudio serológico mediante SAT, entre los años 2000 y $2005(n=38)$. Se caracterizó el patrón clínico, los antecedentes epidemiológicos y la repuesta al tratamiento antimicrobiano mediante la revisión de fichas clínicas o por contacto directo con los médicos tratantes.

Los pacientes se subdividieron en dos grupos: $\mathrm{Pa}$ cientes con brucelosis aguda, definida como síndrome febril y compromiso del estado general, sin foco aparente al examen físico, con hemocultivos positivos para Brucella sp o serología positiva por SAT en títulos $\geq 1 / 160^{9}$ o en los que hubo mejoría clínica con el tratamiento antimicrobiano específico. Pacientes con brucelosis crónica, definida como síndrome febril prolongado de más de 6 meses de evolución ${ }^{15}$ y alguna complicación (lesiones supurativas ósea, en el bazo o hígado) con histología compatible a infección granulomatosa no tuberculosa, con antecedentes epidemiológicos y respuesta a la terapia. Cuando estuvo disponible, se registraron los siguientes exámenes de laboratorio para evaluar si estaban alterados: hemoglobina (valor normal $>12 \mathrm{mg} / \mathrm{dl}$ en mujeres y > $14 \mathrm{mg} / \mathrm{dl}$ en hombres), leucocitos (valor normal 4.000 a $10.000 \mathrm{~mm}^{3}$ ), baciliformes (valor normal 0 a 4\%), VHS (valor normal 1 a $19 \mathrm{~mm}$ a la hora), PCR (valor normal 0 a $0,9 \mathrm{mg} / \mathrm{dl}$ ), SGOT (valor normal 9 a 25 U/L), SGPT (valor normal 7 a $30 \mathrm{U} / \mathrm{L}$ ), GGT (valor normal 4 a $50 \mathrm{U} / \mathrm{L}$ ), fosfatasas alcalinas (valor normal 30 a $100 \mathrm{U} / \mathrm{L}$ ), bilirrubinemia total (valor normal 0 a $1 \mathrm{mg} / \mathrm{dl}$ ), bilirrubinemia directa (valor normal 0 a 0,2 mg/dl) y LDH (valor normal 135 a $225 \mathrm{U} / \mathrm{L})$.

El grupo control estaba constituido por pacientes en los cuales se descartó una brucelosis, con SAT y hemocultivos negativos para Brucella sp y en los que se confirmó algún otro diagnóstico.

Los sueros de ambos grupos fueron analizados mediante Brucellacapt ${ }^{\circledR}$ y ELISA IgM e IgG, por los métodos que se describen a continuación.

Seroaglutinación en tubo $(S A T)^{16}$ : realizada mediante dilución en tubo, utilizando antígenos comerciales de B. abortus (Cenogenics ${ }^{\circledR}$, USA). Las reacciones de aglutinación se leyeron luego de 48 horas de incubación a $37^{\circ} \mathrm{C}$ en baño María. Luego de finalizado el tiempo de incubación, se observó la aglutinación usando una fuente de luz indirecta contra un fondo negro; el título es la dilución máxima del suero que presenta $50 \%$ de las bacterias aglutinadas y el sobrenadante moderadamente turbio, considerándose positivo cuando éste es $\geq 1 / 160^{9}$. En caso de resultado positivo, se repitió la prueba, agregando a la solución 2-mercapto-etanol a una concentración de 0,05 M para destruir los puentes disulfuros de la molécula de IgM, permitiendo sólo la detección de IgG.

Aglutinación con inmunocaptura (Brucellacapt ${ }^{\circledR}$, Vircell): permite la detección de anticuerpos aglutinantes. La fase sólida posee anticuerpos antihumanos de modo que al agregar diluciones seriadas del suero de los pacientes junto con el antígeno, si existen anticuerpos aglutinantes anti-Brucella en el suero del paciente se producirá aglutinación después de incubar por 24 horas a $37{ }^{\circ} \mathrm{C}$. Se consideró positiva una aglutinación que ocupara casi todo el pocillo en una dilución $>1 / 320$.

ELISA para la detección de anticuerpos IgM e IgG anti-lipopolisacárido de B. abortus cepa S-99 (Vircell): se realizó la prueba de enzimo-inmunoanálisis indirecta para determinar anticuerpos IgG e IgM según las instrucciones del fabricante. La interpretación de los resultados se efectuó mediante el índice de anticuerpos calculado con la fórmula: densidad óptica de la muestra x 10/ densidad óptica del suero cut off x 10. Las muestras con índices $<9$ se consideraron negativas para anticuerpos contra brucela; aquellas con índices $>11$ se consideraron positivas y el rango entre 9 y 11 como indeterminado.

Análisis estadístico. Para analizar las diferencias entre los resultados positivos o negativos de las pruebas serológicas evaluados (SAT, ELISA IgG e IgM y Brucellacapt $\left.{ }^{\circledR}\right)$, se usó la prueba exacta de Fisher. Se consideró significativo un valor $\mathrm{p} \leq 0,05$. La compara- 
ción de los índices de anticuerpos IgG e IgM obtenidos por ELISA y títulos obtenidos por Brucellacapt ${ }^{\circledR}$ entre el grupo de brucelosis y el grupo control se realizó mediante la prueba no paramétrica de Wilcoxon. Se consideró significativo un valor $\mathrm{p} \leq 0,05$.

\section{Resultados}

En el período de estudio se recibieron sueros correspondientes a 38 pacientes. De éstos, 10 pertenecían a pacientes que cumplían los criterios definidos para el diagnóstico de brucelosis, que correspondieron a seis hombres y cuatro mujeres, con edad promedio 53 y 46 años, respectivamente. De los 10 pacientes, siete presentaron brucelosis aguda, con hemocultivos positivos para Brucella sp en tres casos (37,5\%); los otros tres fueron pacientes con brucelosis crónica. Todos tenían antecedentes epidemiológicos de contacto con ganado bovino y consumo de lácteos sin pasteurizar.

El grupo control estuvo constituido por 18 pacientes en los cuales se descartó brucelosis con serología (SAT) y hemocultivos negativos para Brucella sp, sin antecedentes epidemiológicos, y en los que se confirmó otro diagnóstico etiológico como: diverticulitis, vasculitis, enfermedad de Still $(n=2)$, síndrome diarreico, endocarditis infecciosa, complicación post operatoria y artritis séptica esterno-clavicular, entre otros.

Los 10 sueros restantes pertenecían a pacientes que no poseían ficha clínica en nuestra institución; no siendo posible contactar con el médico tratante, fueron excluidos del estudio.

La presentación clínica de brucelosis fue un síndrome febril de más de siete días de evolución en la totalidad de los pacientes, con un promedio de 20 días (rango de 7 a 30 días) para la forma aguda y de dos años para la forma crónica. La mayoría de los pacientes presentó compromiso del estado general, mialgias, cefalea y diarrea. Sólo 38\% tuvo hallazgos positivos al examen físico como hepato-esplenomegalia o linfoadenopatías. En los resultados de laboratorio disponibles, destacaba la presencia de PCR, GGT, SGPT y LDH elevadas en la mayoría de los pacientes: 6/7, 6/7, 5/7 y $5 / 5$, respectivamente.

Los resultados serológicos de los 10 pacientes con diagnóstico de brucelosis se observan en la Tabla 1; destaca que 8/10 fueron positivos para ELISA IgG, 7/10 para Brucellacapt ${ }^{\circledR}$ y 5/10 para ELISA IgM. La asociación de Brucellacapt ${ }^{\circledR}+$ ELISA IgG permitió detectar 9 de los 10 pacientes con brucelosis. El paciente 4, que presentó todas las pruebas serológicas negativas, tenía brucelosis crónica de dos años de evolución y el diagnóstico se confirmó por histología. El otro paciente con brucelosis crónica (paciente 9) tenía una evolución de 120 días con SAT, ELISA IgG y Brucellacapt ${ }^{\circledR}$ positivos y ELISA IgM indeterminada.

Las pruebas SAT, Brucellacap ${ }^{\circledR}$ y ELISA IgG permitieron discriminar, en forma significativa, entre los pacientes con brucelosis y el grupo control (Tabla 2, test exacto de Fisher). No ocurrió lo mismo para ELISA IgM.

Todos los pacientes del grupo control presentaron SAT y Brucellacapt ${ }^{\circledR}$ negativas; para ELISA IgG sólo uno fue indeterminado y para ELISA IgM, dos fueron positivos y uno indeterminado (Tabla 2).

Tanto los títulos de anticuerpos por Brucellacapt ${ }^{\circledR}$ como los índices de anticuerpos IgG por ELISA fueron significativamente más altos en el grupo con brucelosis que en el grupo control. Para los valores de los índices de anticuerpos IgM no hubo diferencia estadísticamente significativa (Figuras 1 a 3).

Tabla 1. Caracterización de los pacientes con diagnóstico de brucelosis y resultados obtenidos por los distintos métodos diagnósticos

\begin{tabular}{|c|c|c|c|c|c|c|c|}
\hline Paciente & Edad (años) & $\begin{array}{l}\text { Brucelosis } \\
\text { aguda/crónica }\end{array}$ & Hemocultivo & SAT* & Brucellacapt ${ }^{\circledR}$ & ELISA IgG & ELISA IgM \\
\hline 1 & 30 & Aguda & B. abortus & + & + & + & + \\
\hline 2 & 75 & Aguda & B. abortus & + & + & + & + \\
\hline 3 & 39 & Aguda & Brucella sp & + & - & + & + \\
\hline 4 & 64 & Crónica & Negativo & - & - & - & - \\
\hline 5 & 50 & Aguda & Negativo & + & + & + & - \\
\hline 6 & 40 & Aguda & Negativo & - & - & + & - \\
\hline 7 & 44 & Aguda & Negativo & - & + & - & - \\
\hline 8 & 70 & Aguda & No disponible & + & + & + & + \\
\hline 9 & 60 & Crónica & No disponible & + & + & + & Indeterminado \\
\hline 10 & 31 & Aguda & No disponible & + & + & + & + \\
\hline
\end{tabular}




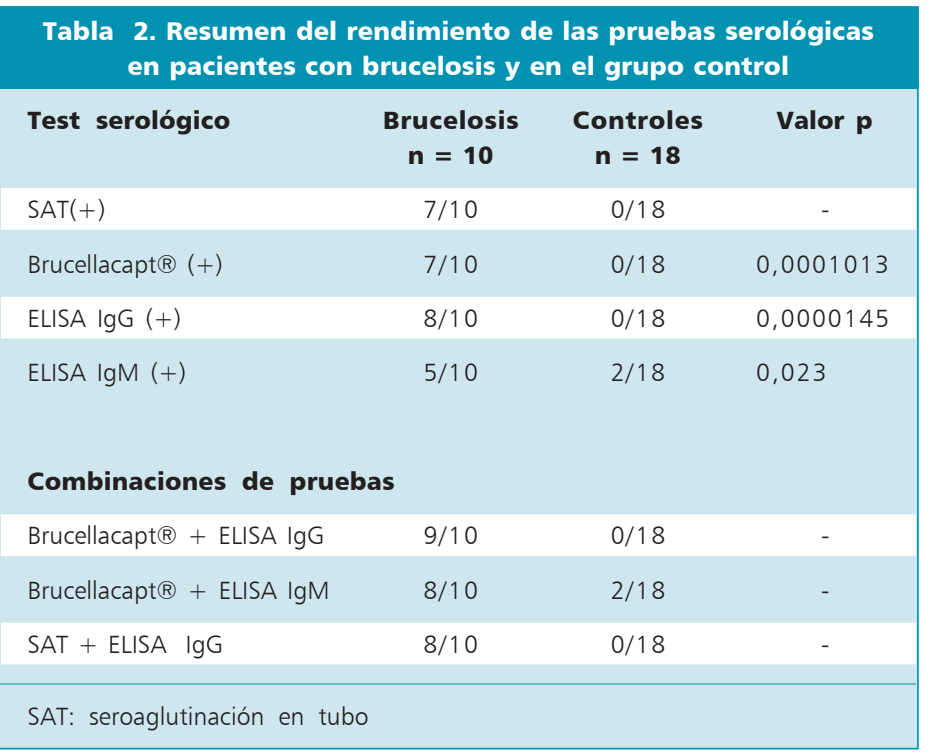

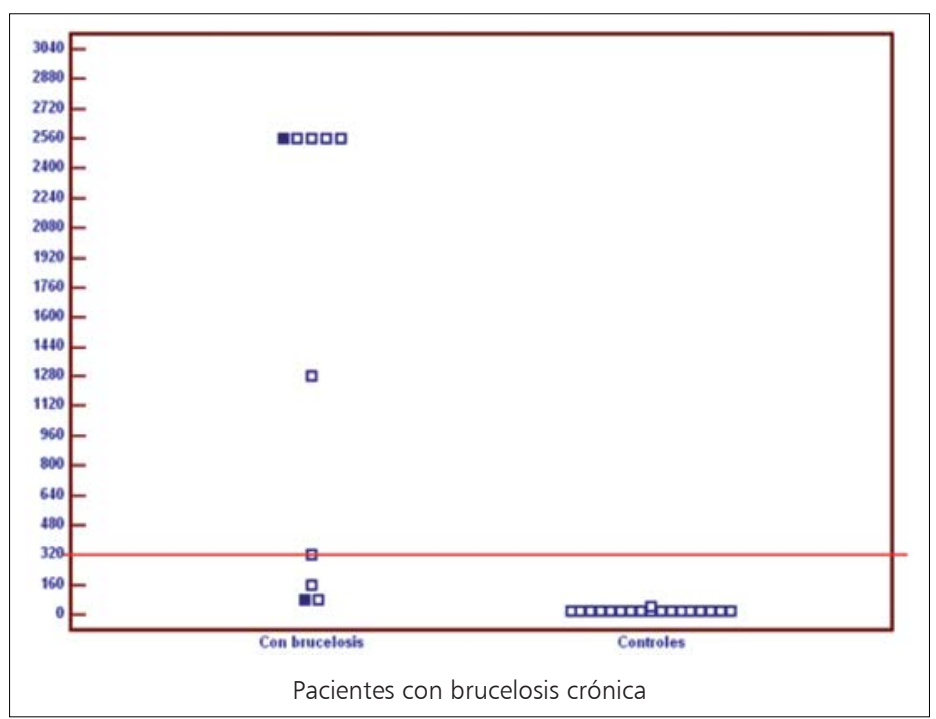

Figura 1. Distribución de los títulos de Brucellacapt ${ }^{\circledR}$ en pacientes con brucelosis crónica y grupo control.

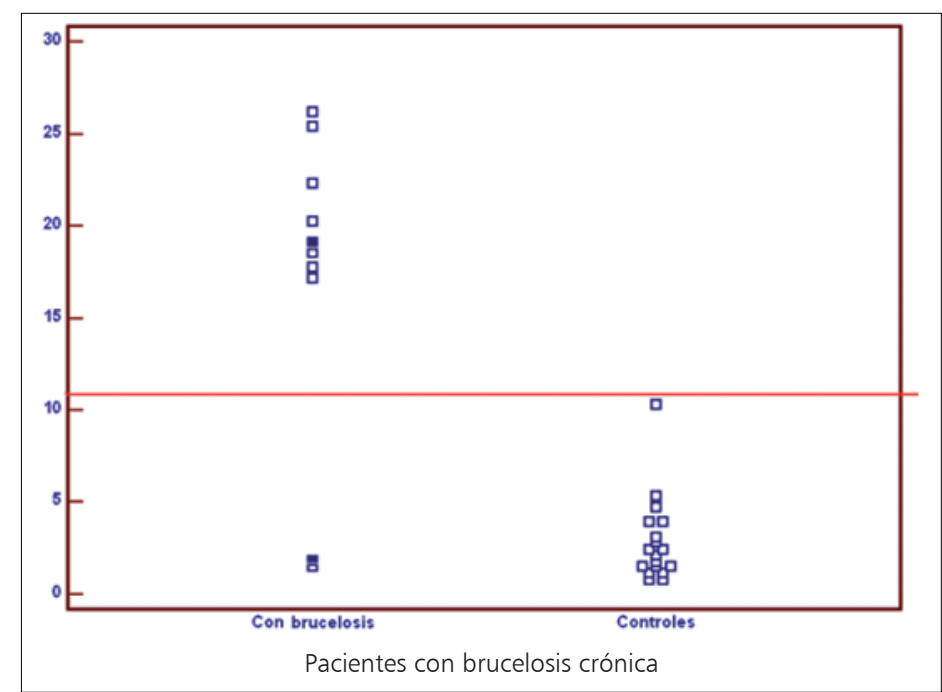

Figura 2. Distribución de índice de anticuerpos IgG determinados por ELISA en pacientes con brucelosis crónica y en el grupo control.

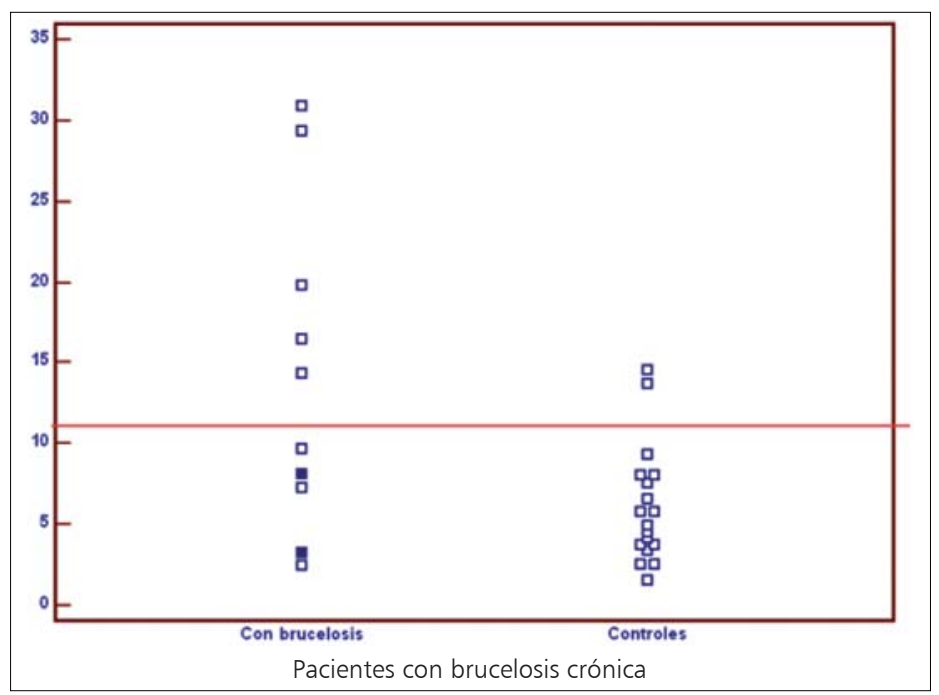

Figura 3. Distribución de índice de anticuerpos lgM determinados por ELISA en pacientes con brucelosis crónica y en el grupo control.

\section{Discusión}

Este trabajo representa la primera evaluación de pruebas comerciales para el diagnóstico de brucelosis humana en nuestro país. Si bien se trata de un estudio retrospectivo con un tamaño de muestra pequeño, se debe considerar que la prevalencia de la enfermedad es baja en la Región Metropolitana y que se reúnen todos los pacientes cuyas muestras se procesaron en el laboratorio de microbiología de la red de Salud UC en un período de 5 años. Por lo anterior, los autores consideran que puede ser un aporte en la información de nuestro país.

En esta serie clínica es importante destacar las manifestaciones gastrointestinales, como diarrea y hepatoesplenomegalia y la alteración de pruebas hepáticas, 
lo cual es concordante con lo descrito por Aziz y cols ${ }^{17}$. Todos los pacientes con brucelosis tenían antecedentes epidemiológicos claros de contacto ocupacional o ingesta de productos lácteos no pasteurizados, por lo que estos datos son de especial importancia en la sospecha diagnóstica. Al igual que lo descrito en la literatura médica, los hemocultivos tienen un rendimiento bajo; Brucella sp es un microoganismo intracelular fastidioso, que crece con dificultad en los medios de cultivo convencionales.

Se encontraron diferencias significativas en el diagnóstico serológico entre el grupo con brucelosis y el grupo control en los índices de IgG por ELISA y los títulos de Brucellacapt ${ }^{\circledR}$, siendo ELISA IgG, por sí solo, mejor que la SAT. Brucellacapt ${ }^{\circledR}$ tuvo un rendimiento menor que el SAT y que ELISA IgG; sin embargo, al combinarlo con ELISA IgG se logró detectar a 9 de los 10 pacientes con diagnóstico de brucelosis, si bien Ciftçi y cols ${ }^{18}$ el año 2005 describió que SAT + ELISA IgG conseguían un mejor rendimiento diagnóstico. Debe tenerse presente que la SAT continua siendo el método de referencia con los inconvenientes mencionados, como el tiempo empleado en su realización, el uso de materiales irritantes al emplear 2 mercapto-etanol, el costo en horas del personal del laboratorio y las reacciones cruzadas con otras bacterias como Escherichia hermanni, Escherichia coli O:157, Salmonella O:30, Stenotrophomonas maltophilia, Vibrio cholerae 01 y Yersinia enterocolitica ${ }^{18}$.

El único paciente que resultó negativo por los cuatro métodos con brucelosis crónica tenía una SAT positiva dos años antes del inicio del estudio (1998), los resultados pudieran explicarse por la remisión de la enfermedad al momento de obtenerse la muestra o a la falta de sensibilidad de las pruebas a títulos bajos, esperables en infección crónica.

La distribución de los índices de IgM por ELISA no presentó diferencia estadísticamente significativa en- tre ambos grupos, con un bajo rendimiento en la detección y con dos falsos positivos en el grupo control, datos coincidentes con la literatura científica ${ }^{19}$.

En conclusión, a pesar del bajo número de muestras estudiadas, estos resultados son similares a los descritos en la literatura médica respecto de la utilidad de la determinación de IgG por ELISA y Brucellacapt ${ }^{\circledR}$ en relación a la SAT, puesto que ambos métodos están comercialmente disponibles, son rápidos y de fácil implementación en los laboratorios del país, con lo que se lograría la estandarización del método.

\section{Resumen}

Introducción: El diagnóstico de brucelosis continúa siendo complejo en la actualidad y se requieren nuevas pruebas de diagnóstico. Objetivo: Evaluar pruebas comerciales para la determinación serológica de anticuerpos anti Brucella sp mediante ELISA e inmunocaptura en una serie clínica de pacientes de la Red de Salud UC. Métodos: Estudio retrospectivo de pacientes cuyos sueros fueron recibidos en el laboratorio de Microbiología para estudio serológico de brucelosis. Se obtuvieron 2 grupos, aquellos que cumplían criterios diagnósticos de brucelosis [cuadro clínico compatible, y/o hemocultivo positivo y/o serología por seroaglutinación en tubo (SAT) en títulos > 1/160] y el grupo control. Todos los sueros se analizaron mediante aglutinación con inmunocaptura (Brucellacapt $\left.{ }^{\circledR}\right)$, ELISA IgM y ELISA IgG. Resultados: De 10 pacientes con brucelosis, los resultados serológicos fueron: 8/10 positivos para ELISA IgG, 7/10 para Brucellacapt ${ }^{\circledR}$ y SAT y 5/10 para ELISA IgM. Discusión: ELISA IgG por si solo fue la mejor prueba para el diagnóstico de brucelosis. La combinación ELISA IgG+ Brucellacapt ${ }^{\circledR}$ alcanza un buen rendimiento de detección (9/10) y puede ser una alternativa a la SAT.

\section{Referencias}

1.- Young E J, Corbel M J. Brucellosis: Clinical and Laboratory Aspects. Boca Ratón CRC Pres Inc Florida, Estados Unidos, 1989; 76-80.

2.- Ergonul O, Celikbas A, Tezeren D, Guvener E, Dokuzoguz B. Analysis of risk factors for laboratory-acquired brucella infections. J Hosp Infect 2004; 56: 223-7.

3.- Lopetegui P. Estrategias y avance del control de la brucelosis bovina en Chile. SAG, 2002. Comunicación personal.

4.- Wall V, Zamorano C G, Paredes L A,
Coyan M, Gómez J C. Prevalencia de brucelosis humana en predios agrícolas ganaderos. Comuna de Puyehue de Osorno X Región, Chile 1999. Rev Chil Salud Pública 2000; 4: 112-6.

5.- Fosgate G T, Carpenter T E, Chomel B B, Case J T, DeBess E E, Reilly K F. Timespace clustering of human brucellosis, California, 1973-1992. Emerg Infect Dis 2002; 8: 672-8.

6.- Chang M H, Glynn M K, Groseclose S L. Endemic, notifiable bioterrorism-related diseases, United States, 1992-1999. Emerg Infect Dis 2003; 9: 556-64.
7.- Ruiz J, Lorente I, Pérez J, Simarro E, Martínez-Campos L. Diagnosis of brucellosis by using blood cultures. J Clin Microbiol 1997; 35: 2417-8.

8.- Memish Z, Mah M, Al Mahmoud S, Al Shaalan M, Khan M. Brucella bacteraemia: clinical and laboratory observations in 160 patients. J Infect 2000; 40: 59-63.

9.- Young E J. Serologic diagnosis of human brucellosis: analysis of 214 cases by agglutination tests and review of the literature. Rev Infect Dis 1991; 13: 359-72. 
10.- Orduña A, Almaraz A, Prado A, Gutiérrez M P, García-Pascual A, Dueñas A, et al. Evaluation of an immunocaptureagglutination test (Brucellacapt ${ }^{\circledR}$ ) for serodiagnosis of human brucellosis.

J Clin Microbiol 2000; 38: 4000-5.

11.- Serra J, Velasco J, Godoy P, Mendoza J. ¿Puede sustituir la prueba de Brucellacapt ${ }^{\circledR}$ a la prueba de Coombs en el diagnóstico de brucelosis humana? Enferm Infecc Microbiol Clin 2001; 19: 202-5.

12.- Smits H L, Abdoel T H, Solera J, Clavijo E, Díaz R. Immunochromatographic Brucellaspecific immunoglobulin $\mathrm{M}$ and $\mathrm{G}$ lateral flow assays for rapid serodiagnosis of human brucellosis. Clin Diagn Lab Immunol 2003; 10: $1141-6$
13.- Araj G F, Lulu A R, Mustafá M Y. Evaluation of ELISA in the diagnosis of acute and chronic brucellosis in human beings. J Hyg (Lond) 1986; 97: 457-69.

14.- Ciftçi C, Oztürk F, Oztekin A, Karaoglan H, Saba R, Gültekin M, et al. Comparison of the serological tests used for the laboratory diagnosis of brucellosis. Mikrobiyol Bul 2005; 39: 291-9.

15.- Ariza J. Brucellosis. Curr Opin Infect Dis 1996; 9: 126-31

16.- Meyer M E. Immune response to brucellae. Manual of Clinical Laboratory Immunology. Washington, DC: American Society for Microbiology 1986; 385-7.

17.- Aziz S, Al-Anazi AR, Al-Aska A I. A review of gastrointestinal manifestations of brucellosis. Saudi J Gastroenterol 2005; 11:
20-7.

18.- Yildiz F, Tanyel E, Hatipoglu C A, Ertem G T, Tülek N, Oral B. Evaluation of Brucella tube agglutination test in patients with brucellosis, patients with bacterial infections other than brucellosis and healthy subjects. Mikrobiyol Bul 2005; 39: 211-7.

19.- Mantecón M A, Gutiérrez P, Zarzosa M P, Dueñas A I, Javier Solera J,

Fernández-Lago L, et al. Utility of an immunocapture-agglutination test and an enzyme-linked immunosorbent assay test against cytosolic proteins from Brucella melitensis B115 in the diagnosis and follow-up of human acute brucellosis. Diagn Microbiol Infect Dis 2006; 55: 27-35. 